\title{
Assessment of Size-Dependent Antimicrobial and Cytotoxic Properties of Silver Nanoparticles
}

\author{
Yoon Jeong, ${ }^{1,2}$ Dong Woo Lim, ${ }^{1,2}$ and Jonghoon Choi ${ }^{1,2}$ \\ ${ }^{1}$ Department of Bionano Technology, Hanyang University, Seoul 133-791, Republic of Korea \\ ${ }^{2}$ Department of Bionano Engineering, Hanyang University ERICA, Ansan 426-791, Republic of Korea \\ Correspondence should be addressed to Jonghoon Choi; jonghchoi@hanyang.ac.kr
}

Received 4 July 2014; Accepted 19 August 2014; Published 15 September 2014

Academic Editor: Protima Rauwel

Copyright (C) 2014 Yoon Jeong et al. This is an open access article distributed under the Creative Commons Attribution License, which permits unrestricted use, distribution, and reproduction in any medium, provided the original work is properly cited.

\begin{abstract}
Nanoscale silver has been increasingly applied to commercial products for their antimicrobial function as antibiotics and disinfectants. In this work, the different sizes of silver nanoparticles (AgNPs) were studied not only in Methylobacterium spp. for their antimicrobial potential but also in human peripheral blood mononuclear cells (PBMCs) for their cytotoxicity in order to determine responses dependent on their particle size. Size controlled silver particles were prepared by chemical reduction of silver cations $\left(\mathrm{Ag}^{+}\right)$and then dispersed in water for their physicochemical characterization using transmission electron microscopy (TEM), dynamic light scattering (DLS), and zeta potential measurements. To ascertain antimicrobial response, water-soluble silver nanoparticles were mixed into Methylobacterium spp. cultured for two days and the sample from the broth was spread on the agar plate for colony counting. $10 \mathrm{~nm}$ nanoparticles showed more antimicrobial activity than $100 \mathrm{~nm}$ particles at which concentrations were equivalently controlled. Increased cytotoxic effect of smaller silver nanoparticles was also observed in PBMCs cocultured with particles. Silver ions released from $10 \mathrm{~nm}$ particles might be correlated with upregulated antimicrobial and cytotoxic properties of AgNPs.
\end{abstract}

\section{Introduction}

Lethal thermal burning and critical infections were a major concern of death in the medieval ages. Since then, silver has been introduced and obtained attention for centuries in health care because of their growth inhibitory capacity against microorganisms. In 1960s, silver sulfadiazine was first used, as an antimicrobial compound, to cure burn patients [13 ] and has also been used for a long time to deal with various diseases like cauterization, pleurodesis, and skin wounds $[1,4,5]$. The silver nitrate solution often called as "Credé's prophylaxis" that is preventive treatment using the instillation of silver nitrate solutions into the conjunctiva of newborns is now regarded as a standard practice in obstetric studies, wound healing applications $[6,7]$, and biomaterial developments $[8,9]$. Because of its fluent antimicrobial action, the number of reported silver usages in the environment as well as in the body has been increasing. Their effect of boosting the number of white blood cells, for example, was demonstrated already [10].
Nanotechnology has opened new and improved modalities of AgNPs in biomedical applications. Compared with macro- or microscale silver materials, nanoscale silver is able to readily release $\mathrm{Ag}^{+}$ions. Before the nanostructured silver is introduced, the applications of silver products have been considerably limited due to their potential side effects which resulted from material defects, impurities, and so forth. Nanotechnology currently is employed as a tool for producing highly purified and well-defined AgNPs, and it has greatly helped to minimize the possible side effects. Enhanced antimicrobial function of AgNPs has promoted active application and commercialization of them in a wide variety of fields and products: catheters, bandages, water filtering, socks, toothpaste, and so forth ([11], see [12] for a database).

Toxicological studies of silver products have been reported actively and scrutinized their safe usages [13-17]. Literatures have been discussing the biological response of AgNPs since year $2000[13,14]$. The essence of those studies is that the size of AgNPs could be an important factor in 
that smaller particles would have larger surface area where it can potentially release more silver ions as a key player for observed toxicity. Also, there is a necessity to demonstrate whether smaller particles have the easier route of endocytosis and exocytosis into cellular membranes and in or out of tissue, blood vessels, and so forth [18-20]. Since the possible mechanisms of the antibacterial property of nanoscale silver has not been clearly shown, however, there is an unmet need to address questions; what the safe range of concentration would be applied to animal/human model, and what the key factors would be to kill bacteria efficiently while maintaining their biocompatibility.

Since blood compatibility might be the first requirement for any biomedical device and for commercial products including AgNPs, here, we report assessment of cytotoxicity of AgNPs which interacted with human PBMCs following physicochemical characterization of prepared AgNPs. We cocultured AgNPs with human PBMCs and continuously observed the number of dead cells which resulted from the constant interaction. Since a particle size might be one of the most significant factors affecting particle interactions with cells, we prepared two different sizes of AgNPs (10 and $100 \mathrm{~nm}$ in average diameters) with identical citric acid-terminated surface. Different concentrations of those nanoparticles were evaluated to the PBMCs respectively, and then, the maximum concentration showing biocompatibility to the PBMCs was obtained, empirically. In order to probe antimicrobial function of the AgNPs that have been previously demonstrated for their safety to human blood cells, we introduced particles to the Methylobacterium spp. and assayed the inhibition responses of AgNPs dependent on different sizes and concentration.

\section{Experimental Methods}

2.1. Silver Particles Preparation. Sodium citrate and silver nitrate were obtained from Sigma-Aldrich. AgNPs were prepared by the well-known Turkevich method [21, 22]. A solution of silver nitrate $\left(1.0 \times 10^{-4} \mathrm{M}\right)$ was heated and the droplets of sodium citrate were added to the solution. The reduction of silver ions occurred as monitored by the solution color change to yellow. Less amount of sodium citrate was added $(0.05 \%)$ to the silver nitrate solution to obtain larger particles. The citrate ions act as both a reducing agent and a capping agent resulting in the citrate terminated AgNPs.

2.2. Characterization. The AgNPs were characterized by TEM and DLS measurement. TEM and high-resolution TEM (HRTEM) images were obtained using a JEOL JEM-2100F microscope. The TEM samples were prepared by drying a droplet of the AgNPs suspension on 300 mesh-size carbon film-coated copper grid. The size and zeta potentials of nanoparticle suspension were measured using DLS (Zetasizer Nano, Malvern, Westborough, MA).

2.3. Cytotoxicity Test. The effects of AgNPs on the viability of PBMCs (ATCC co.) were evaluated using a LIVE/DEAD viability/cytotoxicity kit. Human PBMCs were plated in 6 well

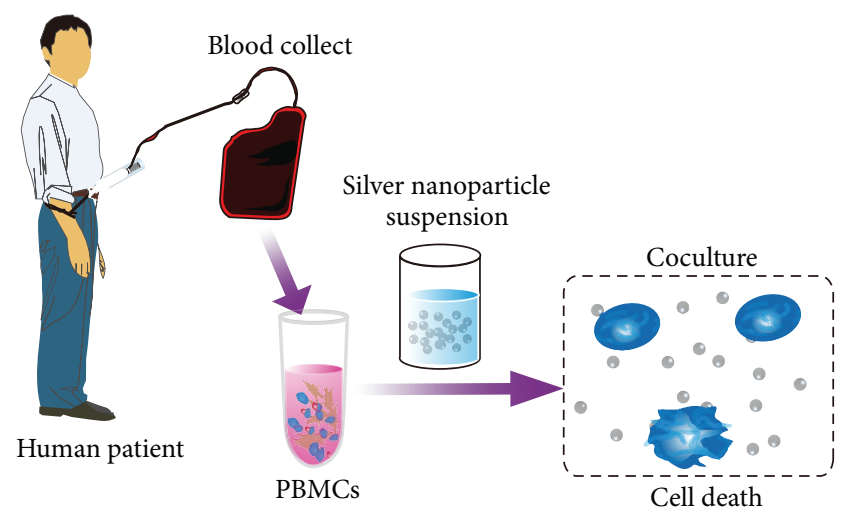

(a)

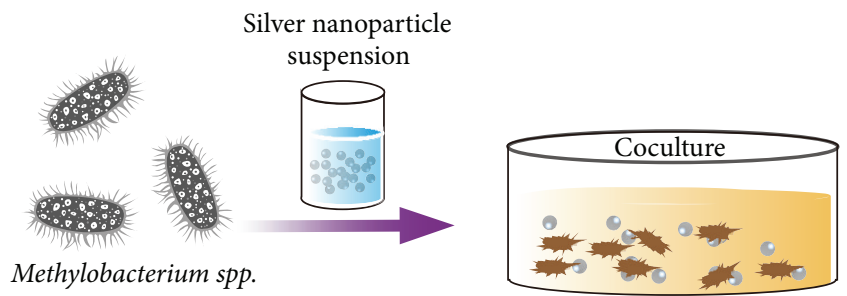

(b)

FIgURE 1: (a) Schematic representation of in vitro cytotoxicity test and (b) antibacterial property assay of AgNPs.

plates $\left(1 \times 10^{5}\right.$ cells per well) in RPMI supplemented with $10 \%(\mathrm{v} / \mathrm{v})$ fetal bovine serum and $1 \%$ sterile antibiotics. The cells were cultured at $37^{\circ} \mathrm{C}$ in a humidified atmosphere of $5 \%$ $\mathrm{CO}_{2} / 95 \%$ air for $8 \mathrm{~h}-12 \mathrm{~h}$. Subsequently, untreated control, DMSO, and 0.1 to $1.0 \mathrm{mg} / \mathrm{mL}$ AgNPs were added to the cells in serum free medium and cultured for 8 hours. Results are quantified as relative values to those of the negative control.

2.4. Antibacterial Tests. The antibacterial activity of the AgNPs was evaluated against Methylobacterium spp. (KCTC 12618 , South Korea) bacteria. A $100 \mu \mathrm{L}$ volume of each cultured bacteria was inoculated in R2A broth (MBcell, U.S.A.) and subsequently incubated with untreated control, methanol, and 0.1 to $1.0 \mathrm{mg} / \mathrm{mL}$ AgNPs. After incubation under shaking conditions (180 r.p.m.) at $30^{\circ} \mathrm{C}$ for 24 hours, $100 \mu \mathrm{L}$ triplicate samples of decimal dilutions were spread on R2A agar (MBcell, U.S.A.) plates. After incubation at $30^{\circ} \mathrm{C}$ for $48 \mathrm{~h}$, the colonies on the plates were counted.

Statistical Analysis. One-tailed Mann-Whitney $U$ test was performed using GraphPad Prism (version 5 for Mac OS X, GraphPad Software, San Diego, CA, USA, http://www.graphpad.com/).

\section{Results and Discussion}

In Figure 1, we showed a schematic drawing of assay procedures for in vitro cytotoxicity and antimicrobial properties of AgNPs. The possible mechanism for cytotoxicity of AgNPs would be that the intact of particles on the cell 

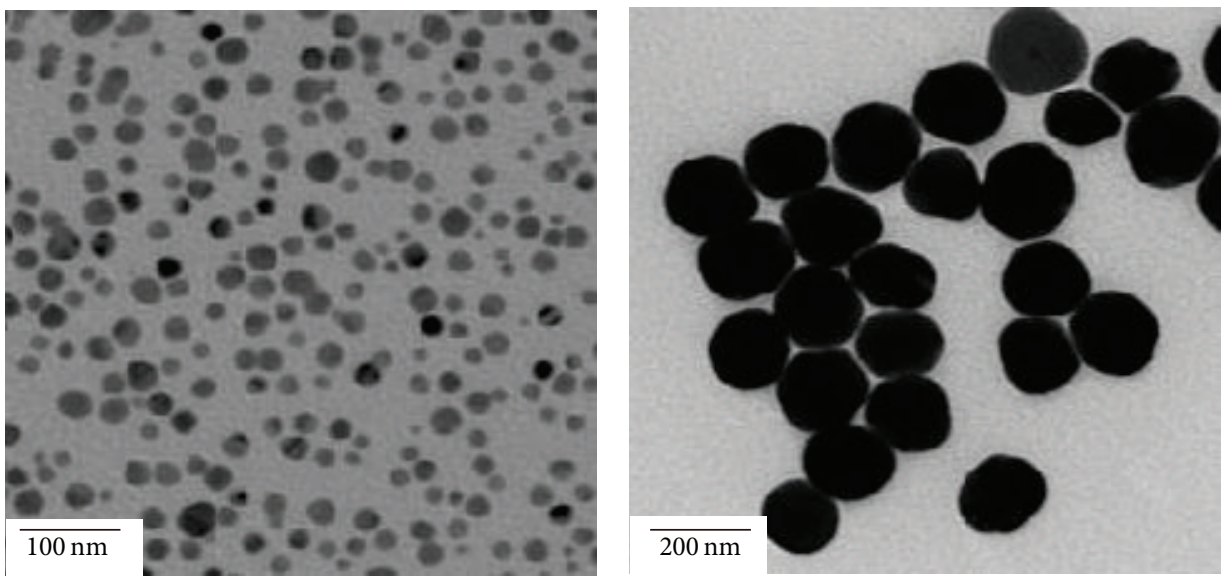

(a)

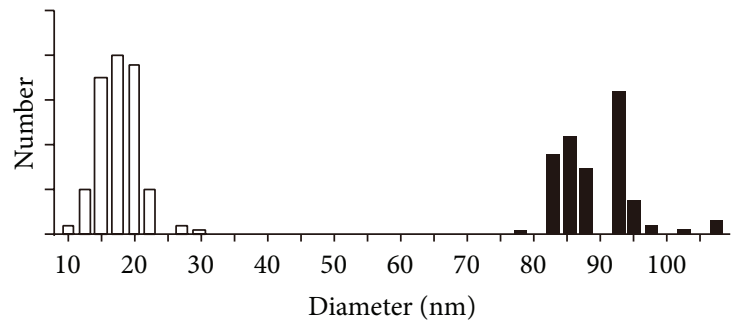

(b)

\begin{tabular}{cccc}
\hline & $\begin{array}{c}\text { Size (TEM) } \\
(\text { diameter, nm) }\end{array}$ & $\begin{array}{c}\text { Size (DLS) } \\
(\text { diameter, nm) }\end{array}$ & $\begin{array}{c}\text { Zeta potential } \\
(\mathrm{mV})\end{array}$ \\
\hline $10 \mathrm{~nm} \mathrm{AgNPs}$ & $14.3 \pm 5.6$ & N/A & $-18.9 \pm 1.1$ \\
$100 \mathrm{~nm}$ AgNPs & $90.8 \pm 19.2$ & $100.2 \pm 11.4$ & $-32.4 \pm 2.7$ \\
\hline
\end{tabular}

(c)

FIgURE 2: Size and shape of AgNPs measured by TEM and DLS (a-c). Comparison of nanoparticle sizes obtained by two different measurement techniques, image analysis of the particles' TEM results and DLS of particle suspension. Smaller nanoparticles ( $<10 \mathrm{~nm})$ can be observed in TEM image analysis results ((a), left) as well as in DLS result ((b), white bars). Lager particles are also shown in a TEM result ((a), right) and in DLS spectra ((b), black bars). (c) Summaries of the physicochemical characteristics of AgNPs tested in the study. Polydispersity indexes of two different size particles were similar at the values of 0.21 for $10 \mathrm{~nm}$ and 0.32 for $100 \mathrm{~nm}$ AgNPs. Size of particles varies depending on the measurement technique applied. Strong negativity of zeta potentials for AgNPs solution is because of the citrates on the surface of them.

wall subsequently ruptured the cell membrane or that silver ions blocked the ion channels of cells. Also, it would be possible that AgNPs and their released silver ions promote denaturation, which cleavages disulfide bonds in cell surfaces, or oxidization of cells. These processes may be responsible for the causes that the cell (or bacteria) loses its control on exposure to the oxidation reactions. Therefore, AgNPs generate reactive oxygen species in water that would degrade the integrity of cell walls.

We prepared different sizes of AgNPs for their effects and each particle was characterized first to compare their differences in physicochemical properties. The size and shape of AgNPs are studied by the TEM. Representative images taken from TEM are shown in Figure 2(a) (Left: $10 \mathrm{~nm}$ AgNPs, Right: $100 \mathrm{~nm}$ AgNPs). Image analysis of TEM taken for nanoparticles reveals the average diameters of two preparations of AgNPs (Figure 2(a)). To confirm the size distribution and mean values observed from TEM, the measurement of particle sizes was also performed in water by using a DLS (Figure 2(b)). Figure 2(c) describes a comparison of nanoparticle sizes between two preparations of AgNPs. Analogous point between two measurement techniques is that they have similar results in particles' mean diameter. However, there are also differences between two methods.
First, smaller particles in nanoparticle suspensions $(<10 \mathrm{~nm})$ may only be seen in TEM. In TEM image analysis, each particle recognized by software can be recorded for their size and registered for their presence. However, in DLS, particles should be able to scatter the light strongly enough to be read by a detector. If the sample is polydisperse, weak light scattering particles (smaller particles) would not be seen by DLS because strong light scattering particles (larger particles) will mask any weak scatterings. Nevertheless, DLS is useful to quickly measure mean size of particles since TEM image analysis usually requires a massive work of image processing. Furthermore, one cannot ignore that the particles in TEM image may not represent the entire samples (biased in a small region). Since each method has its own merits and demerits, one should not rely on the single method of characterization to confirm the mean size of nanoparticles.

In order to study the effects of citrate-covered surface of AgNPs, zeta potentials of each particle were measured in water. The citrated terminated AgNPs would be deprotonated in neutral water carrying enough surface charges to disperse each other. Citrate is a conjugate base of citric acid (2hydroxypropane-1,2,3-tricarboxylic acid) which is a common surfactant for nanoparticles to prevent them from aggregation by providing electrostatic force. It also helps particles to 

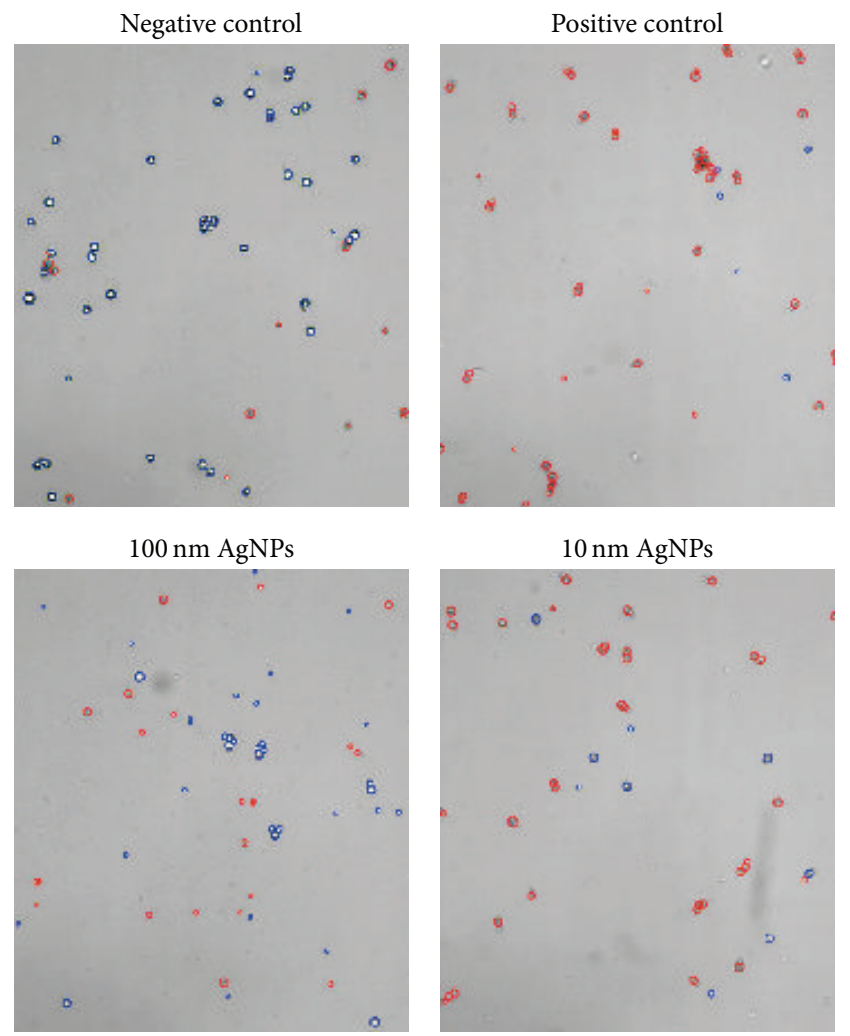

(a)
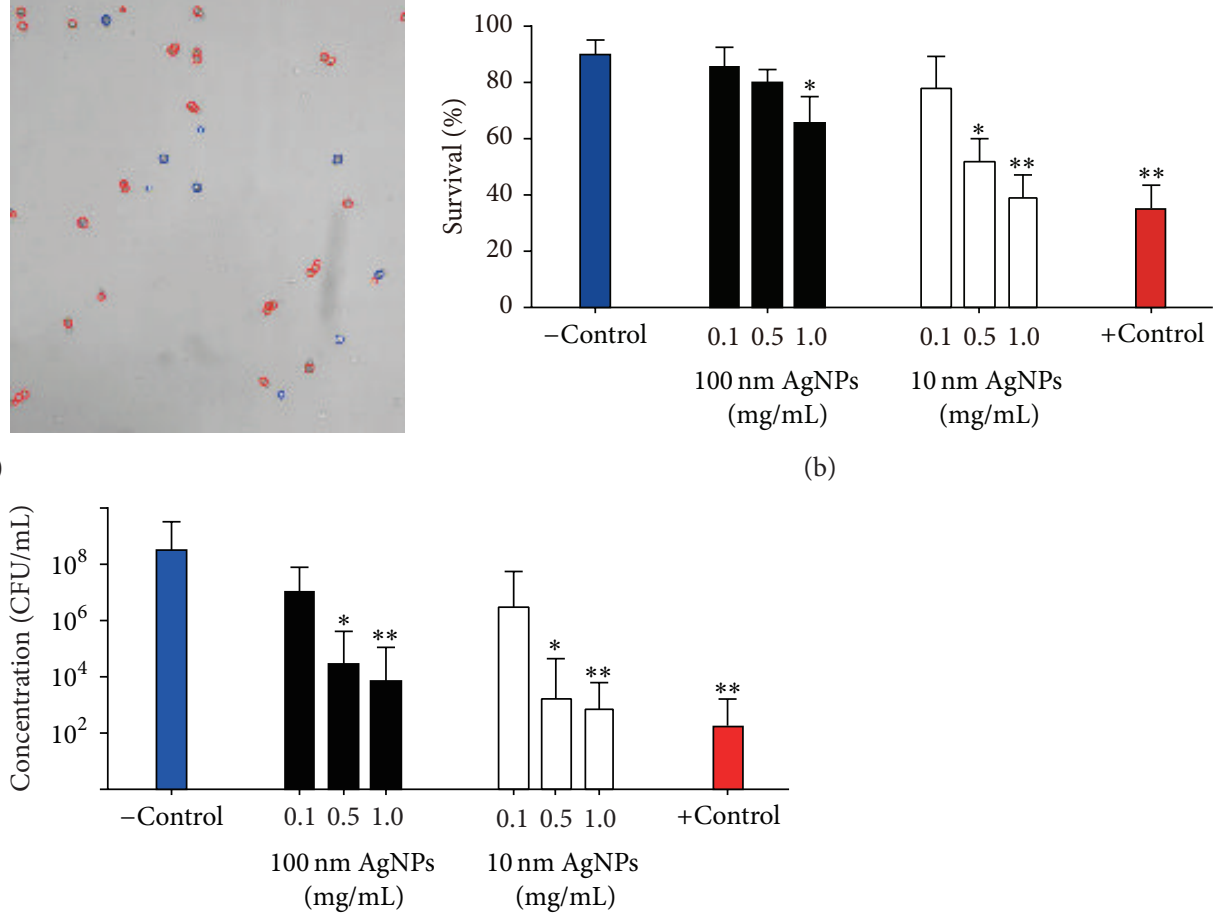

(b)

(c)

FIGURE 3: Cytotoxicity test of AgNPs to PBMCs and antimicrobial effect to Methylobacterium spp. (a) The numbers of live (blue) and dead (red) cells were counted for each sample. (b) $10 \mathrm{~nm}$ AgNPs displayed increased cytotoxicity in $0.5 \mathrm{mg} / \mathrm{mL}$ concentration between two different size particles. $1.0 \mathrm{mg} / \mathrm{mL}$ of $10 \mathrm{~nm}$ AgNPs showed the highest cytotoxicity to PBMCs comparable to the one from the positive control (DMSO). (c) Antimicrobial test of AgNPs against Methylobacterium spp. Both 100 and $10 \mathrm{~nm}$ AgNPs displayed strong antimicrobial effects in $0.5 \mathrm{mg} / \mathrm{mL}$ or higher concentration coincubated with the bacteria. $1.0 \mathrm{mg} / \mathrm{mL}$ of $10 \mathrm{~nm}$ AgNPs showed the equal killing efficiency as to the positive control (methanol). ${ }^{*} P<0.05,{ }^{* *} P<0.01$, one-tailed Mann-Whitney $U$ test.

be dispersed better in water and to protect the core material from degradation or decomposition.

Figure 2(c) summarizes the size and zeta potential values of each sample. The highly negative zeta potential (-18.9 $-32.4 \mathrm{mV}$ ) for both preparations of AgNPs in water is due to citrates on the particle surface deprotonated in neutral water.

Figure 3 summarizes the cytotoxicity and antimicrobial activity caused by AgNPs to human PBMCs and Methylobacterium $s p p$. These results demonstrated that the cytotoxicity was clearly depending on the concentration of nanoparticles added in the cell culture (Figures 3(a) and 3(b)). Antibacterial tests were performed against Methylobacterium spp. with different concentrations of AgNPs (from 0.1 to $1 \mathrm{mg} / \mathrm{mL}$ ) (Figure 3(c)). In the negative control, Methylobacterium spp. have average number of $1 \times 10^{8}$ colonies, while the positive control only contains $4 \times 10^{2}$ colonies. A similar number of colonies as that of the positive control are registered in groups with $1.0 \mathrm{mg} / \mathrm{mL}$ of both 10 and $100 \mathrm{~nm}$ AgNPs. 
Although slightly higher antimicrobial properties of AgNPs are observed in $10 \mathrm{~nm}$ particles, $1.0 \mathrm{mg} / \mathrm{mL}$ AgNPs for both particles is selected as an optimal concentration that can statistically hinder the growth of Methylobacterium spp. as good as the positive control does.

Between two different size preparations of AgNPs, smaller particles (10 $\mathrm{nm}$ average in diameter) showed a higher cytotoxicity when two particles were compared in the same concentration. Smaller particles have a larger surface area when the concentrations of two groups are equally maintained. Large surface area could promote more interactions between particles and cells as well as faster release of silver ions. Citrates on the surface of AgNPs may prevent particles from direct, physical contact with cells in smaller surface area $\left(<10 \mathrm{~cm}^{2}\right)$ but it may not keep up with the increasing silver ion release in larger surface areas $\left(>10 \mathrm{~cm}^{2}\right)$ resulting in that cytotoxicity of $1.0 \mathrm{mg} / \mathrm{mL}$ of $10 \mathrm{~nm}$ AgNPs becomes the highest. Larger surface area also provides higher reactivity of particles and better chances of interacting with solvent ions that will eventually draw more ions from particles.

It has been reported that the silver ions are responsible for AgNPs' antibacterial properties [23-25]. It is an open question whether cytotoxicity happened in an analogous way by the silver ions. Silver ions are able to bind to any sulfhydryl group of the cell wall. Sulfhydryl groups especially in the enzyme systems can be degraded by these silver ion conjugations. Conjugated silver ions can prevent cells from a normal energy and electron transport [23-25]. It is also reported that silver ions can damage bacteria's respiratory chain by conjugating with proteins responsible for it. More studies are required to fully understand whether PBMCs can be lysed by the similar mechanisms that silver ions do for microorganisms.

Further questions regarding the cytotoxicity and antimicrobial property caused by AgNPs are first clarifying which proteins of cells' enzyme system are responsible for conjugation with silver ions. Anion exchanger 1 (AE1) which is transporter of chloride and bicarbonate across plasma membrane and glycophorin C (GYPC) which is the membrane protein of erythrocytes are examples of protein responsible for binding with silver ions released from AgNPs. Another question will be whether other forms of silver (e.g., silver plates) could also cause the similar effects as observed from AgNPs. In order to probe that, systematic study of silver ion release from various morphologies of silver materials should be preceded. In many cases, the metallic silver materials have a poor solubility so that the silver ions released from them could be scarce. However, any new morphology of silver materials introduced by nanotechnology should also be tested for their silver ion release.

In this study, we focused on the role of $\mathrm{Ag}^{+}$ions in antimicrobial and cytotoxic activities but we may further investigate whether the particles by themselves are responsible for a death of PBMCs. Localization of AgNPs inside the cells may answer this issue since PBMCs are notorious for their insufficient cellular systems prepared for an endocytosis. Internalized particles by a membrane rupture would be strong evidence that the PBMCs are lysed by Ag particles. In the same sense, lactate dehydrogenase $(\mathrm{LDH})$ assay would be additional tool for probing cytotoxicity by marking the presence of LDH enzyme diffused out of membrane-degraded PBMCs in the solution with AgNPs. Finally, the observed cytotoxic properties of AgNPs should be carefully analyzed in a scale of either human or small animals' body weights to correctly predict their health risks. Recent studies about AgNPs coated catheters did find a significant release of silver ions in the course of time but the total amount calculated in a body weight was small enough to be negligible for causing a health risk [25].

\section{Conclusion}

Two different size preparations of AgNPs were prepared and assayed for their antimicrobial and cytotoxic properties. $10 \mathrm{~nm}$ AgNPs displayed more cytotoxic and antimicrobial effect compared to $100 \mathrm{~nm}$ sized particles with similar surface chemical composition and the same concentration. A concentration-dependent effect on both cytotoxic and antimicrobial function was observed with substantive killing efficiency occurring at the highest nanoparticle concentrations $(>0.5 \mathrm{mg} / \mathrm{mL})$. These data suggest that the average size of AgNPs is a key factor and blood-contact medical devices and commercial products that contain AgNPs should be carefully monitored for the size and amount applied and their correlating potential safety concerns.

\section{Conflict of Interests}

The authors declare that there is no conflict of interests regarding the publication of this paper.

\section{Acknowledgments}

The authors thank Dr. Taeho Kim at Seoul National University for his assistance with TEM. This research was supported by the Basic Science Research Program through the National Research Foundation of Korea (NRF) funded by the Ministry of Science, ICT, and Future Planning (no. 2008-0061891) and the Basic Science Research Program through the NRF funded by the Ministry of Education (no. 2013R1A1A1012653).

\section{References}

[1] H. J. Klasen, "A historical review of the use of silver in the treatment of burns. II. Renewed interest for silver," Burns, vol. 26, no. 2, pp. 131-138, 2000.

[2] S. Silver, "Bacterial silver resistance: molecular biology and uses and misuses of silver compounds," FEMS Microbiology Reviews, vol. 27, no. 2-3, pp. 341-354, 2003.

[3] B. S. Atiyeh, M. Costagliola, S. N. Hayek, and S. A. Dibo, "Effect of silver on burn wound infection control and healing: review of the literature," Burns, vol. 33, no. 2, pp. 139-148, 2007.

[4] J. Tian, K. K. Y. Wong, C.-M. Ho et al., "Topical delivery of silver nanoparticles promotes wound healing," ChemMedChem, vol. 2, no. 1, pp. 129-136, 2007. 
[5] L. Antonangelo, F. S. Vargas, L. R. Teixeira et al., "Pleurodesis induced by talc or silver nitrate: evaluation of collagen and elastic fibers in pleural remodeling," Lung, vol. 184, no. 2, pp. 105-111, 2006.

[6] C.-S. Chu, A. T. McManus, B. A. Pruitt Jr., and A. D. Mason Jr., "Therapeutic effects of silver nylon dressings with weak direct current on pseudomonas aeruginosa-lnfected burn wounds," Journal of Trauma: Injury Infection \& Critical Care, vol. 28, no. 10, pp. 1488-1492, 1988.

[7] D. Wyatt, D. N. McGowan, and M. P. Najarian, "Comparison of a hydrocolloid dressing and silver sulfadiazine cream in the outpatient management of second-degree burns," Journal of Trauma, vol. 30, no. 7, pp. 857-865, 1990.

[8] T. Bechert, P. Steinrücke, and J.-P. Guggenbichler, "A new method for screening anti-infective biomaterials," Nature Medicine, vol. 6, no. 9, pp. 1053-1056, 2000.

[9] T. Bechert, M. Böswald, S. Lugauer, A. Regenfus, J. Greil, and J.P. Guggenbichler, "The Erlanger silver catheter: in vitro results for antimicrobial activity," Infection, vol. 27, no. 1, pp. S24-S29, 1999.

[10] C. H. Archard and P. E. Weil, "Arch de méd expér \& d'anat path," in Argyria: The Pharmacology of Silver, W. R. Hill and D. M. Pillsbury, Eds., p. 48, The Williams \& Wilkins Company, Baltimore, Md, USA, 1939.

[11] N. Seltenrich, "Nanosilver: weighing the risks and benefits," Environmental Health Perspectives, vol. 121, no. 7, pp. A220A225, 2013.

[12] Project on Emerging Nanotechnologies, Woodrow Wilson International Center for Scholars, 2008, http://www.nanotechproject.org/inventories/silver.

[13] C. Marambio-Jones and E. M. V. Hoek, "A review of the antibacterial effects of silver nanomaterials and potential implications for human health and the environment," Journal of Nanoparticle Research, vol. 12, no. 5, pp. 1531-1551, 2010.

[14] R. de Lima, A. B. Seabra, and N. Durán, "Silver nanoparticles: a brief review of cytotoxicity and genotoxicity of chemically and biogenically synthesized nanoparticles," Journal of Applied Toxicology, vol. 32, no. 11, pp. 867-879, 2012.

[15] G. D. DiVincenzo, C. J. Giordano, and L. S. Schriever, "Biologic monitoring of workers exposed to silver," International Archives of Occupational and Environmental Health, vol. 56, no. 3, pp. 207-215, 1985.

[16] E. J. Jensen, J. Rungby, J. C. Hansen, E. Schmidt, B. Pedersen, and R. Dahl, "Serum concentrations and accumulation of silver in skin during three months treatment with an anti-smoking chewing gum containing silver acetate," Human Toxicology, vol. 7, no. 6, pp. 535-540, 1988.

[17] K. D. Rosenman, A. Moss, and S. Kon, "Argyria: clinical implications of exposure to silver nitrate and silver oxide," Journal of Occupational Medicine, vol. 21, no. 6, pp. 430-435, 1979.

[18] G. A. Sotiriou and S. E. Pratsinis, "Antibacterial activity of nanosilver ions and particles," Environmental Science and Technology, vol. 44, no. 14, pp. 5649-5654, 2010.

[19] S. M. Hussain, K. L. Hess, J. M. Gearhart, K. T. Geiss, and J. J. Schlager, "In vitro toxicity of nanoparticles in BRL 3A rat liver cells," Toxicology in Vitro, vol. 19, no. 7, pp. 975-983, 2005.

[20] A. El-Badawy, D. Feldhake, and R. Venkatapathy, State of the Science Literature Review: Everything Nanosilver and More, Environmental Protection Agency, Washington, DC, USA, 2010, http://goo.gl/GJl0E.
[21] J. Turkevich, P. C. Stevenson, and J. Hillier, "A study of the nucleation and growth processes in the synthesis of colloidal gold," Discussions of the Faraday Society, vol. 11, pp. 55-75, 1951.

[22] J. Turkevich, P. C. Stevenson, and J. Hillier, "The formation of colloidal gold," Journal of Physical Chemistry, vol. 57, no. 7, pp. 670-673, 1953.

[23] J.-P. Guggenbichler, M. Böswald, S. Lugauer, and T. Krall, "A new technology of microdispersed silver in polyurethane induces antimicrobial activity in central venous catheters," Infection, vol. 27, no. 1, pp. S16-S23, 1999.

[24] R. B. Thurman and C. P. Gerba, "The molecular mechanisms of copper and silver ion disinfection of bacteria and viruses," Critical Reviews in Environmental Control, vol. 18, no. 4, pp. 295315, 1988.

[25] D. Roe, B. Karandikar, N. Bonn-Savage, B. Gibbins, and J.B. Roullet, "Antimicrobial surface functionalization of plastic catheters by silver nanoparticles," Journal of Antimicrobial Chemotherapy, vol. 61, no. 4, pp. 869-876, 2008. 

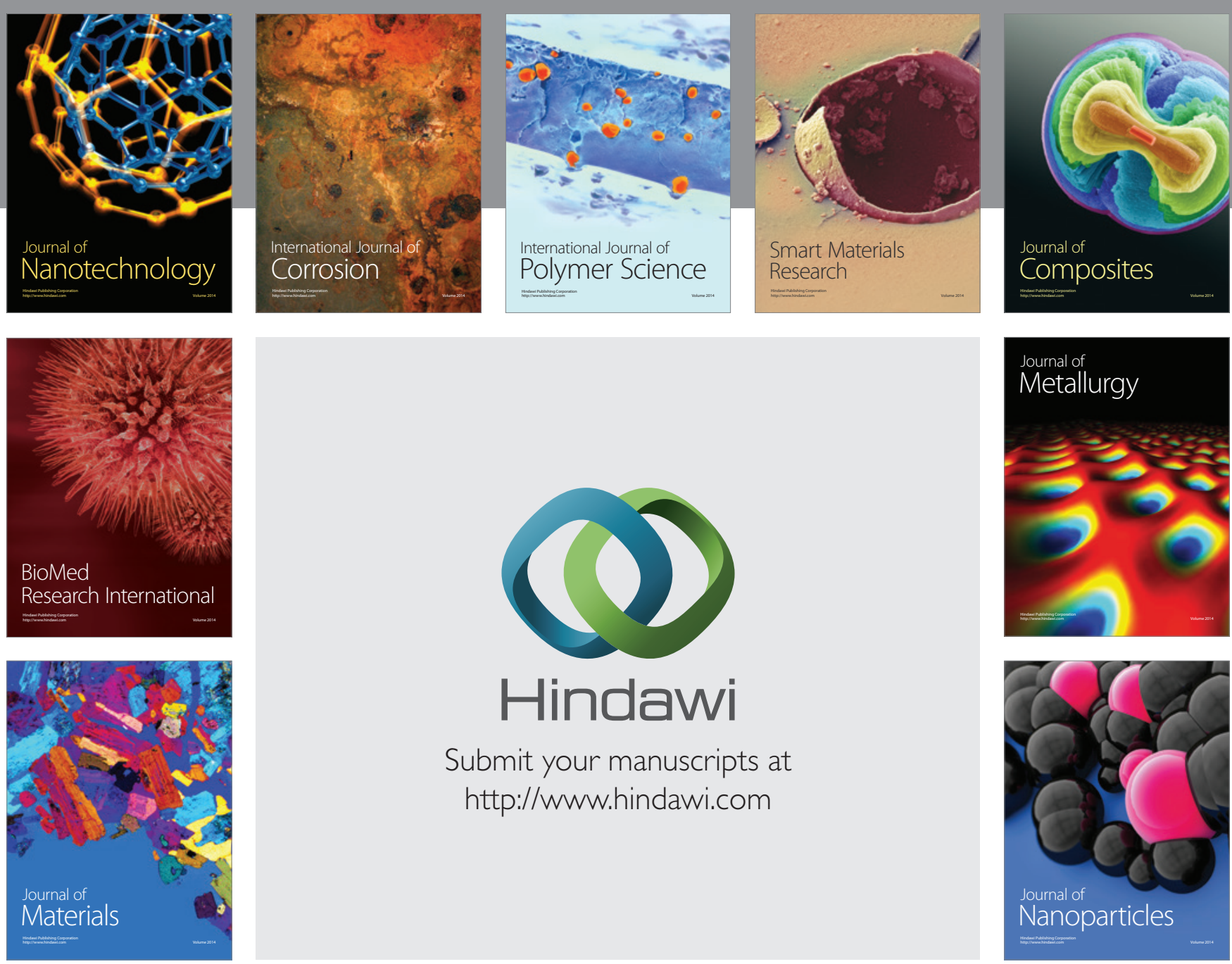

Submit your manuscripts at http://www.hindawi.com
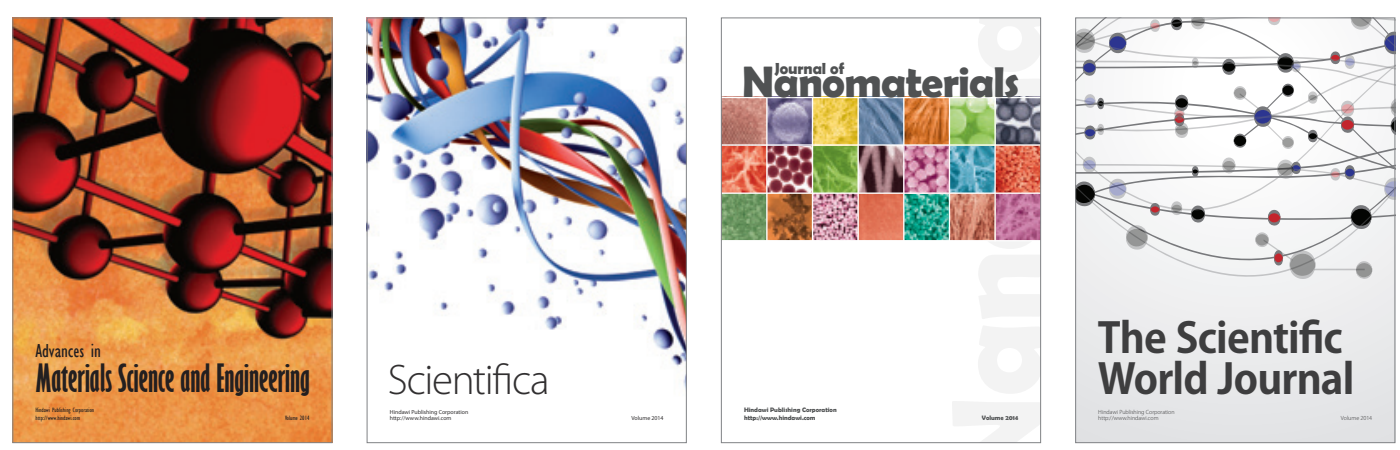

\section{The Scientific World Journal}
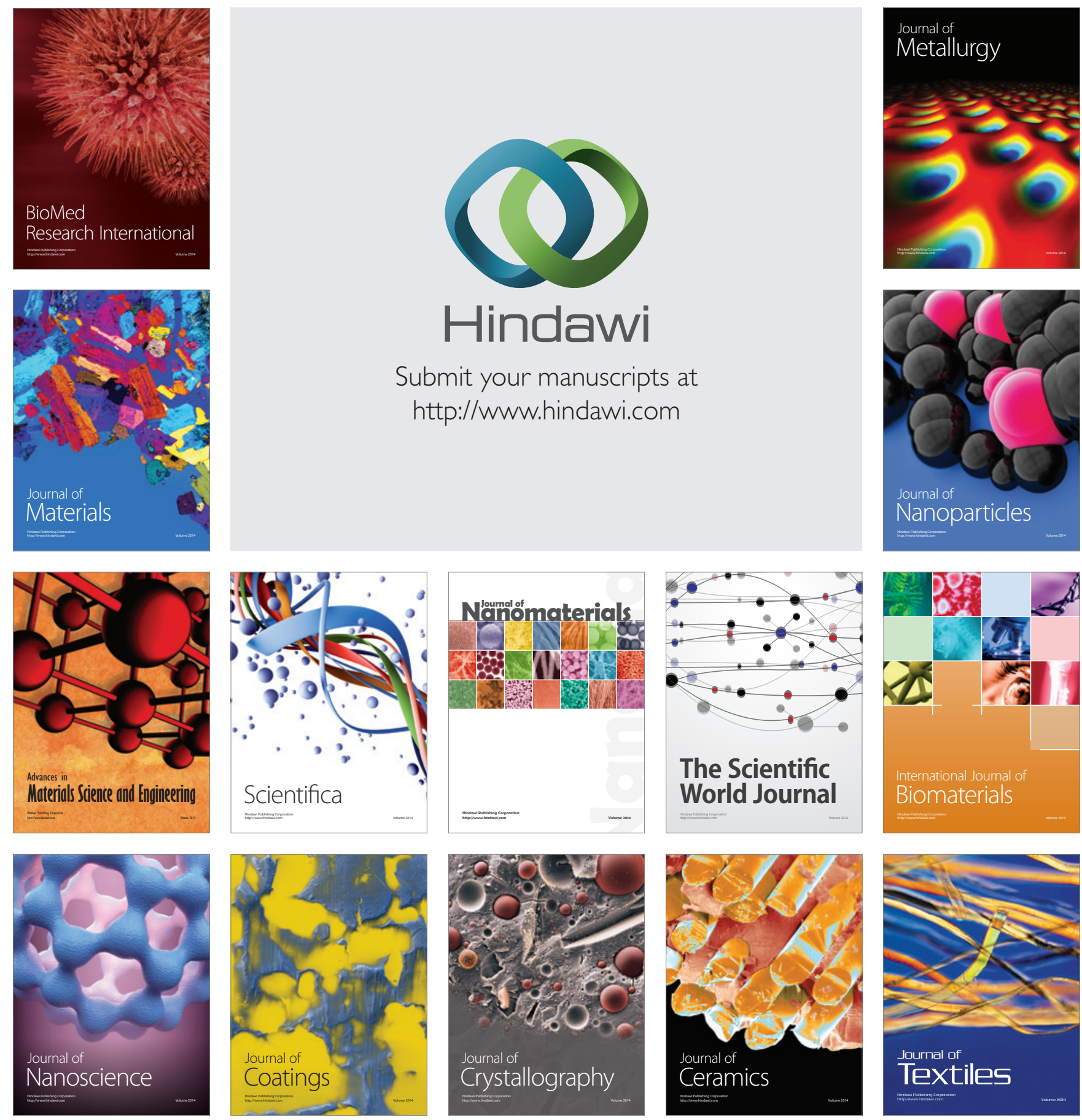